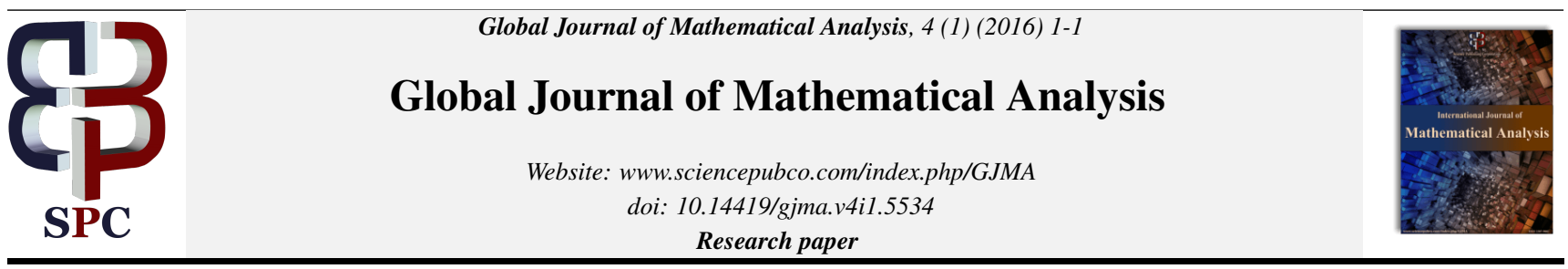

\title{
A simple proof of the closed graph theorem
}

\author{
Alexander G. $\operatorname{Ramm}^{1 *}$ \\ ${ }^{1}$ Mathematics Department, Kansas State University Manhattan, KS 66506, USA \\ ${ }^{*}$ Corresponding author E-mail: ramm@math.ksu.edu
}

\begin{abstract}
Assume that $A$ is a closed linear operator defined on all of a Hilbert space $H$. Then $A$ is bounded. A new short proof of this classical theorem is given on the basis of the uniform boundedness principle. The proof can be easily extended to Banach spaces.
\end{abstract}

Keywords: closed graph theorem; closed linear operator; uniform boundedness principle; new short proof of the closed graph theorem.

\section{Introduction}

We denote by $D(A)$ the domain of definition of $A$, by $A^{*}$ the adjoint operator, by $\|A\|$ the norm of $A$, by $(u, v)$ the inner product in $H$, by $c>0$ various estimation constants are denoted. Let $A$ be a linear operator in $H$ and $u_{n} \in D(A)$. Suppose that $u_{n} \rightarrow u$ and $A u_{n} \rightarrow v$. If the above implies that $u \in D(A)$ and $A u=v$ then the operator $A$ is called closed (see [5]). It is well known (see, [1], [5]) that any bounded sequence in $H$ contains a convergent subsequence.

The following result is classical:

Theorem 1. Let $A$ be a closed linear operator in a Hilbert space $H$, and $D(A)=H$. Then $A$ is bounded.

Theorem 1 is known as the closed graph theorem. Its proof can be found in [1], [5], [7], and in many other texts in functional analysis. These proofs are based on the Baire cathegory theorem. The aim of this note is to give a simple new proof of Theorem 1 using the wellknown uniform boundedness principle, which we state as Theorem 2, and a new result, stated as Theorem 3, which is proved in Section 2 .

In [2] a proof of Theorem 1 is given, which is different from ours. Our proof of Theorem 1 is not only new but also very short.

Proofs of Theorem 2 which are not based on Baire's theorem can be found in [2], problem 27, [3], [4], [6].

Theorem 2. If $\sup _{n}\left|\left(A u_{n}, v\right)\right| \leq \infty$ for every $v \in H$, then $\sup _{n}|| A u_{n} \| \leq \infty$

We assume Theorem 2 known.

The new result we use in the proof of Theorem 1 is the following:

Theorem 3. If $A$ is a linear closed operator with $D(A)=H$, then $D\left(A^{*}\right)=H$.

In Section 2 proofs are given.

\section{Proofs}

\section{Proof of Theorem 3}

If $A$ is a linear closed operator and $D(A)=H$, then $A^{*}$ exists, is closed and densely defined. To prove that $D\left(A^{*}\right)=H$, let $v \in H$ be arbitrary, and $v_{n} \rightarrow v, v_{n} \in D\left(A^{*}\right)$. Let $u \in H$ be arbitrary. Then

$$
\begin{aligned}
& \left(A u, v_{n}\right)=\left(u, A^{*} v_{n}\right) \text { and } \\
& \sup _{n}\left|\left(u, A^{*} v_{n}\right)\right| \leq \sup _{n}\left\|v_{n}|\|\mid A u\| \leq c(u) .\right.
\end{aligned}
$$

By Theorem 2 one has $\sup _{n}\left\|A^{*} v_{n}\right\| \leq c$. Therefore, a subsequence, denoted again $A^{*} v_{n}$, converges weakly in $H: A^{*} v_{n} \rightarrow v^{*}$, and $(A u, v)=\left(u, v^{*}\right)$. Thus, $v \in D\left(A^{*}\right)$, and $D\left(A^{*}\right)=H$ since $v \in H$ was arbitrary.

Theorem 3 is proved.

Proof of Theorem 1. Consider the relation $(A u, v)=\left(u, v^{*}\right)$. Since $D(A)=H$ and $A$ is closed, Theorem 3 says that $D\left(A^{*}\right)=H$, the above relation holds for every $v \in H$, and $v^{*}=A^{*} v$. Suppose that $A$ is unbounded. Then there exists a sequence $u_{n},\left\|u_{n}\right\|=1$, such that

$\left\|A u_{n}\right\| \rightarrow \infty$.

On the other hand, one has:

$$
\sup _{n}\left|\left(A u_{n}, v\right)\right|=\sup _{n}\left|\left(u_{n}, A^{*} v\right)\right| \leq \sup _{n}\left\|u_{n}\right\| \cdot\left\|A^{*} v\right\|=\left\|A^{*} v\right\|:=c(v) .
$$

By Theorem 2 one concludes that $\sup _{n}|| A u_{n} \|<c$. This contradicts (2). Thus, one concludes that $\|A\|<c$. Theorem 1 is proved.

\section{References}

[1] N.Dunford, J. Schwartz, Linear operators, Part I, Interscience, New York, 1958

[2] P. Halmos, A Hilbert space problem book, Springer-Verlag, New York 1974. (problems 52 and 58)

[3] J. Hennefeld, A non-topological proof of the uniform boundedness theorem, Amer. Math. Monthly, 87, (1980), 217.

[4] S. Holland, A Hilbert space proof of the Banach-Steinhaus theorem, Amer. Math. Monthly, 76, (1969), 40-41.

[5] T. Kato, Perturbation theory for linear operators, Springer-Verlag, New York, 1984.

[6] A. Sokal, A relally simple elementary proof of the uniform boundedness theorem, Amer. Math. Monthly, 118, (2011), 450-452.

[7] K. Yosida, Functional analysis, Springer, New York, 1980. 